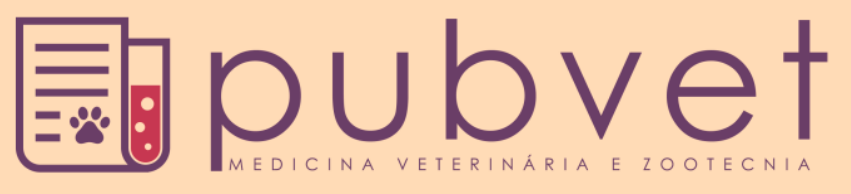

HTTP://DX.DOI.ORG/10.22256/PUBVET.V11N11.1108-1113

\title{
Avaliação parasitológica de excretas de pombos refugiados em edificações da Universidade Federal do Rio Grande do Sul
}

\author{
André Luiz de Souza ${ }^{1}$, Márcia Sueli Scisleski ${ }^{1}$, Sandra Márcia Tietz Marques²* \\ ${ }^{1}$ Medicina Veterinária da Universidade Federal do Rio Grande do Sul. Porto Alegre - RS. E-mail: luiz.souza@ufrgs.br \\ marciasski@gmail.com \\ ${ }^{2}$ Pesquisador, Médico Veterinário, Dr., Laboratório de Helmintoses, Faculdade de Veterinária, Universidade Federal do \\ Rio Grande do Sul. Porto Alegre - RS. \\ *Autor para correspondência: Faculdade de Veterinária, Av. Bento Gonçalves, 9090. Bairro Agronomia, Porto Alegre, RS. \\ CEP: 90540-000. Fax: +5551 3308.7305.E-mail: smtmuni@hotmail.com
}

RESUMO. O objetivo deste estudo foi avaliar a presença de parasitos em fezes de pombos alojados nas edificações do campus do Vale da Universidade Federal do Rio Grande do Sul (UFRGS). Este campus conta com 99 prédios e 85 foram vistoriados. Amostras fecais foram recolhidas de 17 (20\%) deles e o diagnóstico parasitológico se baseou nos métodos de Willis-Mollay (WM), Lutz e Ziehl-Neelsen modificado (ZNm). Por WM e Lutz foram diagnosticados ovos de ascarídeos nas fezes coletadas nos prédios: 1. Departamento de Ecologia; 2. Instituto de Filosofia e Ciências Humanas. Larvas de nematóides foram recuperadas em fezes presentes em seis prédios: 1. Instituto de Informática; 2. Laboratório de Pesquisa do Centro de Biotecnologia; 3. Departamento de Minas da Escola de Engenharia; 4. Biblioteca do Instituto de Física; 5. Instituto de Química - Anfiteatro de Sala de Aula; 6. Laboratório de Informática. Por ZNm, as amostras foram negativas para oocistos de Cryptosporidium spp. As amostras fecais de pombos alojados nas edificações da universidade apresentaram estruturas parasitárias com potencial zoonótico. Conhecer esta diversidade é de fundamental importância para uma melhor gestão do manejo, preservando a saúde das pessoas que transitam pelo campus do Vale da UFRGS.

Palavras chave: Columba livia domestica, fezes, parasito, zoonose

\section{Parasitological evaluation of excretes of pigeons housed in buildings of Federal University of Rio Grande do Sul}

\begin{abstract}
The aim of this study was to evaluate the presence of parasites in feces of pigeons housed in buildings of Campus do Vale of Federal University of Rio Grande do Sul (UFRGS). The campus has 99 buildings and 85 of them were inspected. Fecal samples were collected from $17(20 \%)$ of them and the parasitological diagnosis was based on Willis-Mollay (WM), Lutz and modified Ziehl-Neelsen (ZNm) methods. By WM and Lutz were diagnosed eggs of ascarids in the collected feces: 1. Department of Ecology; 2. Department of Philosophy and Human Sciences. Nematode larvae were recovered from feces present in six buildings: 1. Computing Institute; 2. Research Laboratory of the Biotechnology Center; 3. Mining Department of Engineering School; 4. Library of the Institute of Physics; 5. Chemistry Institute - Classroom Amphitheater; 6. Computing Laboratory. By ZNm, the samples were negative for Cryptosporidium spp. oocysts. The fecal samples from pigeons housed at the university buildings presented parasitic structures with zoonotic potential. Knowing this diversity is of fundamental importance for a better management, preserving the health of people who transit through Campus do Vale UFRGS.
\end{abstract}

Keywords: Columba livia domestica, feces, parasite, zoonosis 


\title{
Evaluación parasitaria de excretas de palomas refugiadas en los edificios de la Universidad Federal de Rio Grande del Sur
}

\begin{abstract}
RESUMEN. El objetivo de este trabajo fue evaluar la presencia de parásitos en heces de palomas alojadas en los edificios del campus del Vale de la Universidad Federal do Rio Grande do Sul (UFRGS). Este campus cuenta con 99 edificios y 85 fueran inspeccionados. Muestras fecales fueron recogidas de 17 (20\%) de ellos y el diagnóstico parasitológico se basó en los métodos de Willis- Mollay (WM), Lutz y Ziehl Neelsen modificado (ZNm). Por los métodos de WM y Lutz fueran identificados huevos de ascarídeos en las heces recogidas en los edificios: 1. Departamento de Ecología; 2 - Instituto de Filosofía y Ciencias Humanas. Larvas de nematodos fueran recuperadas en las heces presentes en seis edificios: 1. Instituto de Informática; 2. Laboratorio de Investigación de lo Centro de Biotecnología; 3. Departamento de Minas de la Escuela de Ingeniaría; 4. Biblioteca del Instituto de Física; 5. Instituto de Química - Anfiteatro de clase; 6. Laboratorio de Informática. Por el método de $\mathrm{ZNm}$, las muestras fueron negativas para oocistos de Cryptosporidium spp. Las muestras fecales de palomas alojadas en los edificios de la Universidad presentaron estructuras parasitarias con potencial zoonótico. Conocer la diversidad de parásitos es de fundamental importancia para una mejor gestión de manejo y preservar la salud de las personas que pasan por el campus del Vale de la UFRGS.
\end{abstract}

Palabras clave: Columba livia domestica, heces, parásito, zoonosis

\section{Introdução}

Pombos estão presentes em quase todos os lugares do planeta devido à capacidade de voar longas distâncias (Clergeau et al., 1998). Sua presença nas cidades é um problema de saúde pública. Por não possuir predadores naturais, sua população cresce muito rápido. São referidos como a principal ameaça para a saúde pública por serem reservatório de pelo menos 70 diferentes microrganismos patogênicos para os seres humanos (Adang et al., 2008, Eslami et al., 2009, Foti et al., 2009).

Columba livia domestica entrou no Brasil no início da colonização portuguesa, oriundo da Europa e da Ásia. É uma ave sinantrópica longeva, vivendo até 16 anos, é granívora e frugívora, porém consome restos de alimentos (Foti et al., 2009). O tamanho da população é influenciado positivamente pelo número de habitantes humanos e pela área da cidade (Hetmański et al., 2011), distribuição espacial dos recursos disponíveis e do fornecimento de alimentos (Rose et al., 2006). Têm papel fundamental na contaminação de fontes de água potável e culturas agrícolas pelas fezes contaminadas, podendo transmitir agentes infecciosos a outras aves (Lillehaug et al., 2005), além de trazer riscos de contaminação para o homem e outros animais (Millán et al., 2004).

Há, também, fatores responsáveis pelo deslocamento das aves para as cidades, como a maior disponibilidade de locais para ninhos, onde a existência de cavidades artificiais pode atrair aves que se utilizam destes lugares. A ocorrência, nestes espaços, de uma concentração maior de espécies vegetais atrativas para aves através de seus frutos e flores, como as plantadas em pomares, podem chamar a atenção de espécies frutívoras e nectarívoras. Além disso, podem se alimentarem de moluscos, helmintos terrestres e insetos, sendo estes últimos, muitas vezes, carreadores de estágios infectantes de helmintos, também hospedeiro alternativo de alguns helmintos de outras aves com quem convivem e são filogeneticamente próximas (Figueiredo, 2007).

Graves problemas de saúde podem afetar os pombos, mas as infecções parasitárias apresentam o papel mais importante, pois estas aves se dispersam e se apossam de locais de criação de espécies domésticas se beneficiando de suas dietas e também de patógenos (Adang et al., 2008). As infecções parasitárias em aves são onipresentes, mesmo quando ocorrem em quantidades baixas, podendo resultar em doenças subclínicas (Badparva et al., 2015). A literatura sobre o conhecimento da fauna parasitária de pombos urbanos se referem àquelas aves que são utilizadas como bioindicadores (Schilderman et al., 1997) ou no cardápio alimentar de humanos, as quais relevam importância das condições sanitárias próprias para consumo humano (Razmi et al., 2007, Adang et al., 2008, Qi et al., 2011, Radfar et al., 2012). 
A fauna parasitária em pombos com relatos no Brasil é escassa e os gêneros descritos são: Acuaria, Capilaria, Ornithostrongylus, Ascaridia, Brachylaemus, Tanaisia e Raillietina (Silva et al., 1990, Marques et al., 2007). No arquipélago das Ilhas Canárias foram detectados, por exames fecais e necropsia, os parasitos Raillietina micracantha, Tetrameres fissispina, Acuaria spiralis, Ascaridia columbae e Aonchotheca sp. (Foronda et al., 2004); enquanto na Nigéria há relato dos gêneros Raillietina, Hymenolepis e Amoebotaenia (Adang et al., 2008); no Irã foram registrados os gêneros Ascaridia, Hadjelia, Cotugnia e Raillietina e oocistos dos gêneros Eimeria e Cryptosporidium (Badparva et al., $\underline{2015}$, Radfar et al., 2012).

O objetivo desta investigação é verificar a ocorrência de helmintos em pombos da espécie
Columba livia domestica que se alojam em edificações n o Campus do Vale da Universidade Federal do Rio Grande do Sul.

\section{Material e Métodos}

O campus do vale da Universidade Federal do Rio Grande do Sul está localizado no bairro Agronomia, município de Porto Alegre (Figura 1) e conta com 99 prédios. Foram vistoriados 85 prédios no ano de 2016 e a metodologia consistiu em recolher as fezes com o auxílio de uma espátula, depositá-las em sacos plásticos, com as análises executadas em até 24 horas no Laboratório de Helmintoses da Faculdade de Veterinária. Este projeto recebeu o número de protocolo de pesquisa do Comitê de Ética no Uso de Animais (CEUA) nº 20487/1.

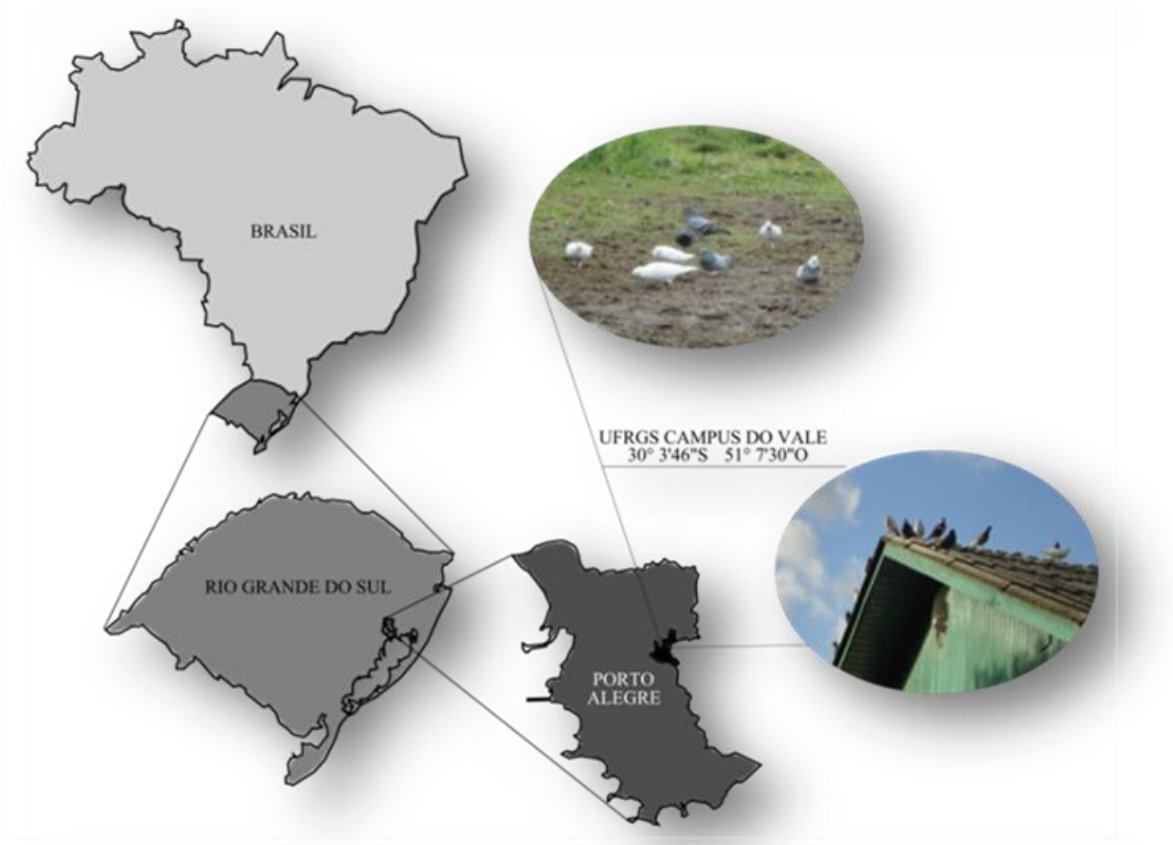

Figura 1 - Localização do Campus do Vale da UFRGS, Bairro Agronomia, Porto Alegre, Rio Grande do Sul, Brasil.

O diagnóstico parasitológico se baseou em três técnicas: Willis-Mollay (WM), Lutz e ZiehlNeelsen modificado (ZNm), respectivamente, flutuação com solução saturada de cloreto de sódio (densidade $=1,20$ ), sedimentação espontânea em água e esfregaço fecal corado com fucsina fenicada. Cinco gramas de fezes maceradas foram processados para os métodos de WM e Lutz. O material sobrenadante obtido pela técnica de WM foi imediatamente examinado entre lâmina e lamínula, adicionando-se uma gota de lugol em microscópico óptico. Após sedimentação por 12 horas, pela técnica de Lutz, parte do sedimento foi colocado em lâmina de vidro, adicionado lugol e examinado em microscopia de luz. Para a pesquisa de Cryptosporidium spp. foi utilizada a técnica de $\mathrm{ZNm}$, os esfregaço corados foram observados em microscopia de imersão (Hoffmann, 1987).

Com base nos dados mensais de abundância média obtidos por observação visual, as aves foram classificadas em quatro categorias segundo Ramírez Albores et al. (2002): 1. Abundante (total de 40 ou mais Indivíduos registrados); 2. Comum (17 a 39 indivíduos registrados); 3. Escasso (11 a 16 indivíduos registrados); 4. Irregular (cinco a 10 indivíduos registrados) e 5. Raros (um a quatro indivíduos registrados). 


\section{Resultados e Discussão}

Durante o ano de 2016, as áreas investigadas apresentaram, na maioria, registro de pombos com média classificada como escasso. Os pombos circulam entre prédios, árvores e espaços abertos e com frequência são vistos junto as lixeiras e em locais com disponibilidade de alimentos. As fezes estavam depositadas em aparelhos de ar condicionado, parapeitos de janelas, canos e redes de proteção, preferencialmente (Figura 2). Constatou-se que 20\% (17/85) dos prédios vistoriados apresentaram fezes de pombos, são eles: Centro de Biotecnologia; Departamento de Materiais, Laboratório de Ensaios e Modelos Estruturais, Departamento de Minas e Metalurgia da Escola de Engenharia; Departamento de Ecologia; Departamento de Biofísica e Centro de Biotecnologia; Instituto de Física; Departamento Paleontologia e Museu Paleontológico do Instituto de Geociências; Laboratório de Pesquisa, salas de Físico-Química, do Departamento de Química Inorgânica e Biblioteca do Instituto de Química; Biblioteca do Instituto de Física; Administração, Secretaria e Depto. de Pós-Graduação de Instituto de Filosofia e Ciências Humanas; Centro de Estudos de Geologia Costeira e Oceânica do Instituto de Geociências; Departamento de PósGraduação do Instituto de Biociências; Salas de aula e Biblioteca do Instituto de Matemática e Física; e salas de aula do Laboratório de Informática.

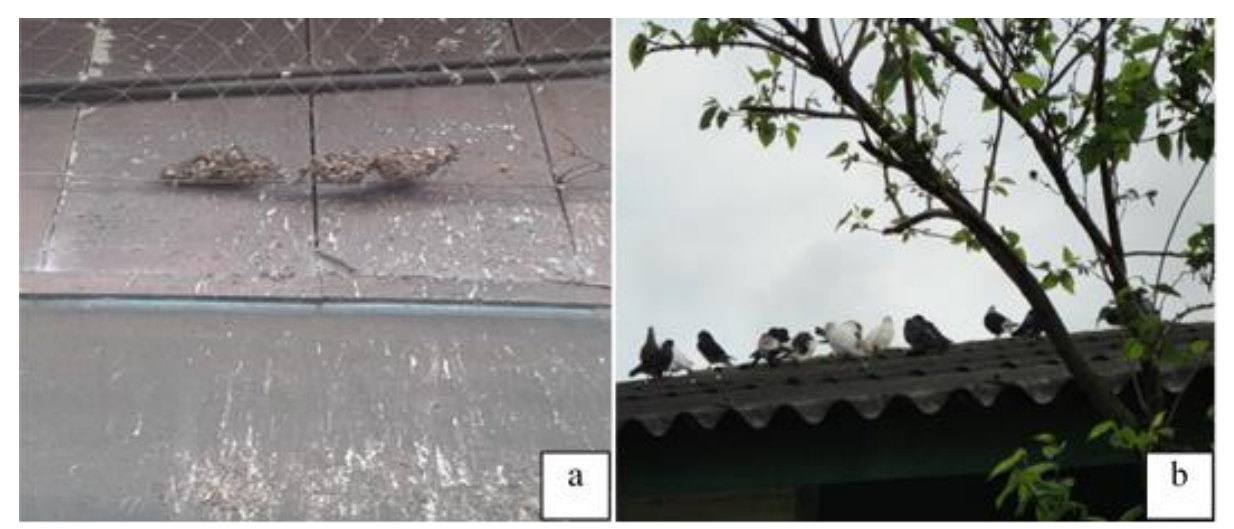

Figura 2 - a. Fezes em rede de proteção e parede suja; b. aves no telhado.

Foram diagnosticados: a) ovos de ascarídeos (Figura 3a) nas fezes coletadas no prédio do Departamento de Ecologia do Instituto de Biociências e no prédio do Instituto de Filosofia e Ciências Humanas (IFCH); b) grande quantidade de ácaros vivos nas fezes coletadas no IFCH e no Instituto de Informática; c) grande quantidade de larvas (Figuras 2b e 2c) em seis prédios (Instituto de Informática, Laboratório de Pesquisa do Centro de Biotecnologia, Departamento de Minas da Escola de Engenharia, Biblioteca do Instituto de Física, Instituto de
Química, Anfiteatro de sala de aula e Laboratório de Informática). Todos os esfregaços fecais foram negativos para a técnica de $\mathrm{ZNm}$. As fezes nas quais foram detectados ovos de ascarídeos foram submetidas à coprocultura por até 40 dias, sem sucesso na obtenção de larvas, o que impossibilitou a identificação em nível de gênero. Quanto às larvas detectadas, também não foi possível a diferenciação de gêneros por análise morfológica em microscopia óptica, sendo factível por metodologias moleculares, porém o alto custo inviabilizou.

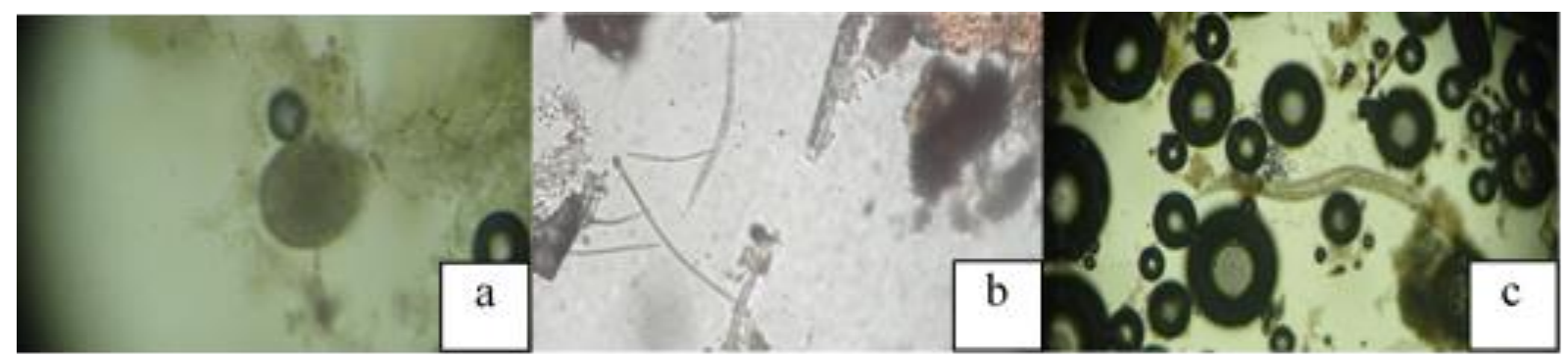

Figura 3 - Ovos de ascarídeos (a) e larvas de nematoides (b, c) recuperadas pelos métodos de WM e Lutz em fezes de Columba livia domestica alojadas no Campus do Vale da UFRGS, em 2016. 
Foram executados esfregaços fecais para a identificação de oocistos de Cryptosporidium spp., causador da criptosporidiose, doença importante em aves e com registro em mais de 30 espécies no mundo (Ryan et al., 2003, Ryan, 2010) cuja preocupação na investigação foi devido à característica zoonótica, também pela facilidade de dispersão caso os pombos estejam albergando oocistos. De igual modo, a presença de ovos de ascarídeos é preocupante; entretanto não foi possível a evolução dos ovos para a fase larvária. Sabe-se que ascarídeo tem grande potencial zoonótico, cujos ovos são resistentes aos desinfetantes e condições do meio ambiente, além de responsáveis pela Larva Migrans Visceral.

Excrementos de pássaros encontrados em qualquer lugar devem ser considerados formas de dispersão de patógenos zoonóticos, como fungos e estruturas parasitárias, relativamente resistentes no meio ambiente e que nas fezes encontram meio propício a sua manutenção. A maioria das fezes coletada estava secas o que facilita a sua dispersão. Os pombos em cidades exploram diferentes tipos de recursos para sua dieta variável como restos alimentares produzidos pelo homem e alguns tipos de sementes de interesse econômico, como soja, trigo e milho, embora sua preferência seja por sementes de plantas silvestres (Ranvaud et al., 2001). Felizmente a universidade vem tomando medidas preventivas regularmente, como a colocação de telas, limpeza periódica de parapeitos nos prédios, evitando acúmulo de lixo e de material orgânico. Estas ações são importantes pois o número de pessoas que transitam no Campus do Vale é muito relevante, representado, por estudantes de graduação, cujas vagas oferecidas/ano são de 4.358; contando, por exemplo, com 30.102 alunos de graduação matriculados somente no segundo semestre de 2015. Além destes, professores, técnicos e pessoal terceirizado. A universidade atua, também, em projetos sociais envolvendo, por exemplo, em 2015 um efetivo de 8631 visitantes, além de projetos culturais que beneficiaram 83.313 pessoas na Universidade Federal do Rio Grande do Sul.

Outro aspecto importante diz respeito à colonização de cidades por espécies sinantrópicas como pombos e roedores. Nas cidades ocorrem várias situações ambientais favoráveis que levam a uma verdadeira explosão demográfica de populações de animais e infecções transmitidas. A multiplicidade de nichos ecológicos em ambiente urbano estimula o aumento de certas espécies animais como consequiência de temperaturas ótimas para reprodução e crescimento, bem como a abundância de alimentos e água. Esta situação promove a superlotação e o aumento de indivíduos da mesma espécie, criando um problema ao induzir à emergência e propagação de novos patógenos e alergias. A adaptação dos pombos ao habitat urbano e a colonização de novos nichos ecológicos levaram à acumulação de uma grande quantidade nas praças, monumentos e edifícios e em todos os locais utilizados para a construção de ninhos e depósito de fezes, representando um risco biológico para a saúde de seres humanos e animais, uma vez que pode ser uma fonte de infecções virais, bacterianas e fúngicas, bem como um substrato para ectoparasitos (Tarsitano et al., 2010).

\section{Conclusão}

As amostras fecais de pombos alojados nas edificações da universidade apresentaram estruturas parasitárias com potencial zoonótico. Conhecer esta diversidade é de fundamental importância para uma melhor gestão do manejo preservando a saúde das pessoas que transitam pelo campus do Vale da UFRGS.

\section{Referências Bibliográficas}

Adang, K. L., Oniye, S. L., Ajanusi, O. J., Ezealor, A. U. \& Abdu, P. A. 2008. Gastrointestinal helminths of the domestic pigeons (Columba livia domestica gmelin, 1789 aves: columbidae) in Zaria, northern Nigeria. Science World Journal, 3, 33-37.

Badparva, E., Ezatpour, B., Azami, M. \& Badparva, M. 2015. First report of birds infection by intestinal parasites in Khorramabad, west Iran. Journal of Parasitic Diseases, 39, 720-724.

Clergeau, P., Savard, J.-P. L., Mennechez, G. \& Falardeau, G. 1998. Bird abundance and diversity along an urban-rural gradient: a comparative study between two cities on different continents. Condor, 100, 413-425.

Eslami, A., Ghaemi, P. \& Rahbari, S. 2009. Parasitic infections of free-range chickens from Golestan Province, Iran. Iranian Journal of Parasitology, 4, 10-14.

Figueiredo, L. F. Aves na cidade. 2007. Disponível em <http://www.ib.usp.br/ceo/parqu/avesnacid.ht $\underline{\mathrm{m}>}$. Acesso em: 11 abril 2017.

Foronda, P., Valladares, B., Rivera-Medina, J. A., 
Figueruelo, E., Abreu, N. \& Casanova, J. C. 2004. Parasites of Columba livia (Aves: Columbiformes) in Tenerife (Canary Islands) and their role in the conservation biology of the laurel pigeons. Parasite, 11, 311-316.

Foti, M., Daidone, A., Aleo, A., Pizzimenti, A., Giacopello, C. \& Mammina, C. 2009. Salmonella bongori 48: z35:-in Migratory Birds, Italy. Emerging Infectious Diseases, 15, 502-503.

Hetmański, T., Bocheński, M., Tryjanowski, P. \& Skórka, P. 2011. The effect of habitat and number of inhabitants on the population sizes of feral pigeons around towns in northern Poland. European Journal of Wildlife Research, 57, 421-428.

Hoffmann, R. P. 1987. Diagnóstico de parasitismo veterinário. Sulina, Porto Alegre, Brasil.

Lillehaug, A., Jonassen, C. M., Bergsjø, B., Hofshagen, M., Tharaldsen, J., Nesse, L. L. \& Handeland, K. 2005. Screening of feral pigeon (Colomba livia), mallard (Anas platyrhynchos) and graylag goose (Anser anser) populations for Campylobacter spp., Salmonella spp., avian influenza virus and avian paramyxovirus. Acta Veterinaria Scandinavica, 46, 193-202.

Marques, S. M. T., Quadros, R. M., Silva, C. J. \& Baldo, M. 2007. Parasites of pigeons (Columba livia) in urban areas of lages, Southern Brazil. Parasitología latinoamericana, 62, 183-187.

Millán, J., Aduriz, G., Moreno, B., Juste, R. A. \& Barral, M. 2004. Salmonella isolates from wild birds and mammals in the Basque Country (Spain). Revue Scientifique et Technique, 23, 905-912.

Qi, M., Wang, R., Ning, C., Li, X., Zhang, L., Jian, F., Sun, Y. \& Xiao, L. 2011. Cryptosporidium spp. in pet birds: genetic diversity and potential public health significance. Experimental Parasitology, 128, 336-340.

Radfar, M. H., Asl, E. N., Seghinsara, H. R., Dehaghi, M. M. \& Fathi, S. 2012. Biodiversity and prevalence of parasites of domestic pigeons (Columba livia domestica) in a selected semiarid zone of South Khorasan, Iran. Tropical Animal Health and Production, 44, 225-229.

Ramírez Albores, J. E., Cedillo, R. \& Guadalupe, M. A. 2002. Avifauna de la región oriente de la sierra de Huautla, Morelos, México. Anales del Instituto de Biología. Serie Zoología, 73, 91-
111.

Ranvaud, R., Freitas, K. C., Bucher, E. H., Dias, H. S., Avanzo, V. C. \& Alberts, C. C. 2001. Diet of Eared Doves (Zenaida auriculata, Aves, Columbidae) in a sugar-cane colony in South-eastern Brazil. Brazilian Journal of Biology, 61, 651-660.

Razmi, G. R., Kalidari, G. A. \& Maleki, M. 2007. First report of the Hadjelia truncata infestation in pigeons of Iran. Iranian Journal of Veterinary Research, 8, 175-177.

Rose, E., Nagel, P. \& Haag-Wackernagel, D. 2006. Spatio-temporal use of the urban habitat by feral pigeons (Columba livia). Behavioral Ecology and Sociobiology, 60, 242-254.

Ryan, U. 2010. Cryptosporidium in birds, fish and amphibians. Experimental Parasitology, 124, 113-120.

Ryan, U. M., Xiao, L., Read, C., Sulaiman, I. M., Monis, P., Lal, A. A., Fayer, R. \& Pavlasek, I. 2003. A redescription of Cryptosporidium galli Pavlasek, $1999 \quad$ (Apicomplexa: Cryptosporidiidae) from birds. Journal of Parasitology, 89, 809-813.

Schilderman, P. A., Hoogewerff, J. A., van Schooten, F.-J., Maas, L. M., Moonen, E. J., Van Os, B. J., van Wijnen, J. H. \& Kleinjans, J. 1997. Possible relevance of pigeons as an indicator species for monitoring air pollution. Environmental health perspectives, 105, 322330.

Silva, C. C., Mattos Júnior, D. G. \& Ramires, P. M. 1990. Helmintos parasitas de Columba livia (Gm) no Município de Säo Gonçalo, Rio de Janeiro. Arquivo Brasileiro de Medicina Veterinária e Zootecnia, 42, 391-394.

Tarsitano, E., Greco, G., Decaro, N., Nicassio, F., Lucente, M. S., Buonavoglia, C. \& Tempesta, M. 2010. Environmental monitoring and analysis of faecal contamination in an urban setting in the city of Bari (Apulia region, Italy): health and hygiene implications. International Journal of Environmental Research and Public Health, 7, 3972-3986.

Article History:

Received 30 June 2017

Accepted 29 July 2017

Available on line 6 September 2017

License information: This is an open-access article distributed under the terms of the Creative Commons Attribution License 4.0, which permits unrestricted use, distribution, and reproduction in any medium, provided the original work is properly cited. 